\title{
The role of GABA in detecting visual motion
}

\author{
Martin Egelhaaf, Alexander Borst and Birgit Pilz \\ Max-Planck-Institut für biologische Kybernetik, Tübingen (F.R.G.)
}

(Accepted 7 November 1989)

Key words: Vision; Motion detection; Direction selectivity; Shunting inhibition; Fly; $\gamma$-Aminobutyric acid

\begin{abstract}
The basic computations underlying the extraction of motion from the visual environment have been characterized in great detail. A non-linear interaction, such as a multiplication, between neighbouring visual elements was shown to be the core of biological motion detectors in different species ranging from insects to man. GABA ( $\gamma$-aminobutyric acid)-ergic inhibitory synapses suppressing the responses to motion in one direction but not in the other are widely accepted to be the cellular basis for this non-linear interaction. Based on model predictions we can show in combined pharmacological and electrophysiological experiments that in the fly motion detection system GABAergic synapses do not play this role but rather are involved in another important step of motion computation. This makes a reconsideration of the role of inhibition in other motion detection systems necessary.
\end{abstract}

The analysis of motion plays a central role in biological vision systems. The direction and velocity of a moving object, however, are not explicitly encoded at the level of the retinal input. Instead, they have to be computed from the time-dependent brightness fluctuations of the retinal image in a series of processing steps. There is good evidence in several biological vision systems ranging from insects to different vertebrate species including man in favour of a particular class of motion detection mechanisms, the so-called correlation-type of movement detectors $^{7,21,22}$, or mathematically equivalent versions of them $^{1,23}$. Roughly speaking, this type of movement detector has two spatially separated input channels which interact in a non-linear way after one of the signals has been delayed. Various formal operations were proposed for this non-linear interaction. Examples are a logical gate as in the well-known Barlow-Levick scheme ${ }^{4}$, a summation followed by a threshold operation ${ }^{14}$, or simply a multiplication as in the eldest and most widely discussed scheme of this class of movement detection model $^{21,22}$. It is obvious that a motion detection unit built in this way is directionally selective to some extent. However, under realistic conditions it would also respond to correlated input signals which are independent of the direction of motion, such as fluctuations of the mean light intensity ${ }^{9,23}$. This problem can be alleviated in a simple way by composing the detector of two such units, mirror-symmetrically arranged and subtracted from each other (Fig. 1 left). Since the motion-independent response components are identical in both subunits, they are eliminated by the subtraction stage ${ }^{9}$. Thus direction selectivity of a movement detector is considerably enhanced, if it is computed by a two-stage process consisting of a non-linear interaction and a subsequent subtraction stage.

Which are the cellular mechanisms responsible for the different operations in the formal movement detector model? To account for the non-linear interaction between the two detector input channels, various cellular models have been proposed ${ }^{14,26,27}$. The so-called shunting-inhibition model is the most popular one among neurophysiologists. This cellular model relies on the non-linear interaction of an excitatory and an inhibitory, GABA ( $\gamma$-aminobutyric acid)-ergic synapse which receive their input from neighbouring locations in visual space. To some extent, it approximates a multiplication-like interaction $^{14,27}$. During motion in the detector's preferred direction, signals are transmitted by the excitatory synapse, while they are suppressed during motion in the opposite direction. This mechanism owes its popularity to the finding that direction selectivity of motion-sensitive cells in the retina $a^{2,3,8,29}$ and the visual cortex ${ }^{25}$ of various vertebrate species as well as in the fly ${ }^{24}$ is greatly reduced by application of GABA antagonists. This has been interpreted as an interference with the essential nonlinear interaction of the detector input channels and, thus, in favor of the shunting inhibition model ${ }^{2,16,24}$. This interpretation, however, is only conclusive, if direction selectivity is acquired in a single processing step, i.e. just by the non-linear interaction of the movement detector

Correspondence: M. Egelhaaf, Max-Planck-Institut für biologische Kybernetik, Spemannstraße 38, D-7400 Tübingen, F.R.G. 
input channels. It may be not cogent in case of a two-stage process such as shown in Fig. 1, since then direction selectivity is not only reduced by disturbance of the non-linear interaction but also when the subtraction process is impaired by GABA antagonists. This suggests that direction selectivity on its own does not represent a good indicator for the performance of the movement detector's non-linear processing stage.

To decide between these alternatives, i.e. effects of GABA antagonists on the non-linear interaction vs subtraction, additional information is required which is more specific than direction selectivity. If the non-linear interaction can be approximated sufficiently well by a multiplication and no other non-linearities distort the detector input signals significantly, this information can be derived from Fourier analysis of local movement detector responses (Fig. 1 right). When a grating with

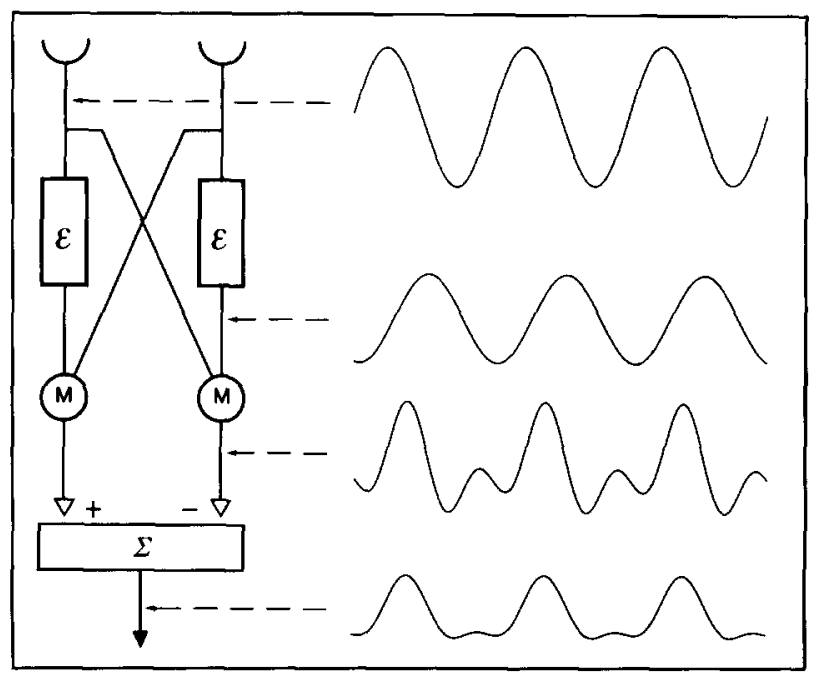

Fig. 1. Correlation-type of movement detector and its response to a grating with sinusoidally modulated brightness moving from the left to the right. A detector in its simplest form consists of two mirror-symmetrical subunits. In each subunit the signal carried by one input channel is delayed in some way (indicated by $\varepsilon$ ), such as by a low-pass filter, and then interacts non-linearly with the instantaneous signal of the neighbouring input channel. The final detector output is given by the difference of the subunit outputs. The model simulations of the detector signals as displayed on the right hand side are based on two assumptions: (i) the non-linear interaction stage is given by a multiplication; (ii) there are no non-linearities prior to the movement detector which significant!y distort the time course of the input signals. The two signals simultaneously arriving at the multiplication stage (shown here for the right detector subunit) are then sinusoids and the result of their multiplication consists of only the fundamental and second harmonic frequency component of the temporal frequency of the stimulus. After subtracting the two detector subunits the amplitude of the second harmonic decreases and may completely vanish in case of mathematically perfect subtraction. For the model simulations shown here, it has been assumed that the two detector subunits are not exactly balanced and that the subunit which contributes to the overall response of the detector with a negative sign has the smaller gain $(0.9)$. sinusoidally modulated brightness passes a movement detector with a constant velocity, the signals converging at the multiplication stage should also be sinusoidally modulated in time. The resulting responses of the detector subunits are then modulated with the fundamental frequency and the second harmonic of the input signal ${ }^{9.14}$. After subtracting the subunits' signals from each other, the second harmonic is less pronounced in the final detector output and may even disappear ${ }^{9}$. Since the second harmonic frequency component is produced by the multiplication process and reduced by the subtraction stage (Fig. 1 right), it can be used to decide at which processing stage of a movement detector GABA plays its

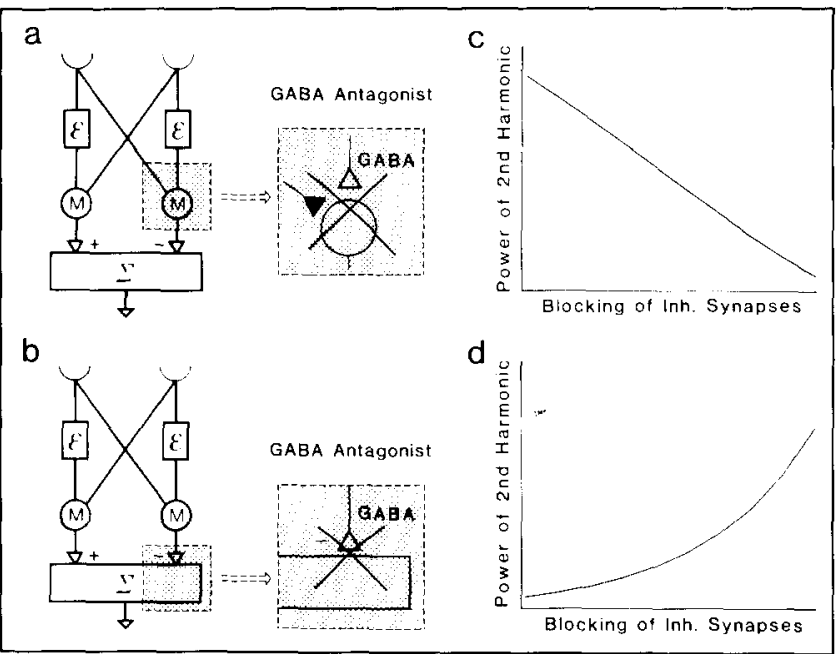

Fig. 2. Predicted consequences of a pharmacological impairment of either the multiplication $(a, c)$ or the subtraction $(b, d)$ stage of the movement detector. a: the neuronal realization of the multiplication-like interaction is assumed to be represented by the interaction of an excitatory and a GABAergic inhibitory synapse as proposed by Torre and Poggio ${ }^{27}$. Blocking of this synapse by a GABA antagonist should gradually reduce the power of the second harmonic frequency component of the movement detector response (c). $\mathrm{b}$ : the negative input to the subtraction stage of the formal movement detector model is assumed to correspond to a GABAergic inhibitory synapse in a neuronal implementation of the detector. If this synapse is blocked by a GABA antagonist, the power of the second harmonic frequency component in the output signal of the movement detector is predicted to increase (d). Hence interference with either the multiplication or the subtraction stage leads to qualitatively different predictions with respect to the contribution of the second harmonic frequency component to the overall movement detector response. The model simulations were based on the equation of an electrical equivalent circuit of biological membranes $V(t)=\left(g_{\mathrm{e}} E_{\mathrm{e}}+g_{\mathrm{i}} E_{\mathrm{i}}+g_{1} E_{\mathrm{l}}\right) /\left(g_{\mathrm{c}}+g_{\mathrm{i}}+g_{1}\right) . g_{\mathrm{c}}, g_{\mathrm{i}}$ and $g_{1}$ denote the conductance of the excitatory and GABAergic inhibitory synapses as well as the leak conductance, respectively. $E_{\mathrm{e}}, E_{\mathrm{i}}$ and $E_{1}$ denote the corresponding batteries. If the non-linear interaction is to be represented by this equation, the following numerical values were chosen: $E_{\mathrm{e}}=-10 \mathrm{mV}, E_{\mathrm{i}}=E_{1}=-70 \mathrm{mV}$; see ref. 27 . In case of the subtraction stage, the numerical values of the different batteries in this equation amounted to: $E_{\mathrm{e}}=-10 \mathrm{mV}, E_{\mathrm{i}}=-70 \mathrm{mV}, E_{1}=-50$ $\mathrm{mV}$. The conductances $g_{\mathrm{e}}$ and $g_{\mathrm{i}}$ were assumed to be proportional to the corresponding input signals of the synapses; $g_{1}$ was constant. $c$ and $d$ : the inhibitory synapses were weighted by a gain factor which was varied in the simulation between 1 and 0 . 
critical role (Fig. 2). If inhibitory GABAergic synapses are the basis of the non-linear interaction of the detector input channels, the power of the second harmonic frequency response component is expected to decrease after blocking the GABAergic synapses by an antagonist (Fig. 2a). This prediction is not affected if, in addition to the non-linear interaction, also the subtraction stage is based on GABAergic synapses and blocked pharmacologically. However, if GABAergic synapses are responsible for the subtraction process alone the second harmonic frequency component should increase (Fig. 2b).

These predictions were tested in the motion detection system of the fly which proved to be an excellent model system for studying various visual information processing tasks ${ }^{6.10}$. In particular, its motion detection system can be described satisfactorily by the formal scheme outlined above: it computes motion in a two-stage process with a multiplication-like interaction of the detector input channels and a separate subtraction stage ${ }^{9}$. In the third visual ganglion of the blowfly Calliphora erythrocephala we recorded the responses of a directionally selective motion-sensitive interneurone, the H1-cell, which can be identified individually in different preparations ${ }^{15}$. This cell has an extended receptive field and spatially integrates the output signals of large retinotopic arrays of local movement detectors. Since the local movement detectors all look at a different spatial phase of the stimulus pattern, their response modulations are expected to be phase-shifted in time. Spatial integration then eliminates the temporal modulations of the local detector responses. We prevent spatial integration by presenting the motion stimulus to the fly's eye through a sufficiently small vertical slit. In this way local detector responses can be derived from spatially integrating visual interneurones 9 . When a sinewave grating is moved behind the slit one obtains, as predicted for a multiplication-like movement detector non-linearity, periodic responses to motion in both the cell's preferred and null direction which are modulated in time. The temporal modulations consist of almost only the fundamental and second harmonic frequency component ${ }^{9 *}$ (Fig. 3). In the present study we combine the slit stimulation technique with injection of picrotoxinin into the fly's haemolymph next to the third visual ganglion. Picrotoxinin is known to be a non-competitive blocker of inhibitory chloride channels, especially, though not exclusively, of those activated by $\mathrm{GABA}^{5}$. After a short delay of about $5-20$ $\mathrm{s}$ the responses, on average, change dramatically for some 20-60 min before returning again to their preinjection shape. Most notably, the power of the second harmonic frequency increases by about a factor of 10 . Moreover, there is also a pronounced increase in the mean spike rate during motion in both the preferred and null direction resulting in a reduction of direction selectivity. The latter feature was already reported before and interpreted as evidence for the shunting inhibition model $^{24}$. According to our model predictions, however, the concomitant increase of the second harmonic frequency component following picrotoxinin injection suggests that in the fly visual system GABA is involved in the subtraction and not in the multiplication process of motion detection.

Whether this holds also true for other systems where GABA was found to impair direction selectivity cannot yet be decided. Other criteria than were used here may be required for distinguishing between an elimination of the non-linear interaction stage and the subtraction stage in two cases: (i) if the non-linear interaction cannot be approximated sufficiently well by a multiplication, or (ii) if sinusoidal movement detector input signals are distorted by additional non-linearities. Some experimental evidence, in addition to the pharmacological one, has been adduced in favour of a direct involvement of inhibitory processes in the non-linear interaction stage in the retina of various vertebrate species ${ }^{4,16,18,28.29}$. None of these studies, however, considers the significance and possible involvement of a subtraction stage, separate from the non-linear interaction, in generating direction selectivity. Interestingly, there are specific features commonly found in the responses of directionally selective cells to apparent motion stimuli which suggest that both these processes may be involved in computing direction selectivity: during apparent motion in the null direction, suppression of the responses is frequently observed in directionally selective cells of the retina ${ }^{4}$ and lateral geniculate nucleus ${ }^{17}$ of the rabbit, the cat striate corte $x^{11}$ ${ }^{13}$, as well as in area MT of monkeys ${ }^{19}$. In many of these cells, however, also an enhancement of the responses during apparent motion in the cell's preferred direction can be detected ${ }^{11,13,19.20}$; this is even true for the rabbit retina as is revealed by the seminal paper of Barlow and Levick $^{4}$. It is obvious that an enhancement and a suppression in a single cell can hardly be explained by a single-stage process of motion detection based on a

\footnotetext{
* A second harmonic frequency component could alternatively arise, if the input signals are half-wave rectified and processed in separate onand off-detectors, the outputs of which are then summated to form the final detector response. This kind of preprocessing of the movement detector input has recently been proposed ${ }^{12}$. Since the on- and off-detectors act in counterphase when stimulated by motion of sinewave gratings, the final response is expected to contain a strong second harmonic as long as the detector responds to motion at all. Moreover, the relative contribution of the second harmonic and the fundamental frequency should be more or less invariant with respect to the direction of motion as well as the strength of the subtraction stage. This, however, is in contrast to our experimental data (see Fig. 3 and ref. 9).
} 


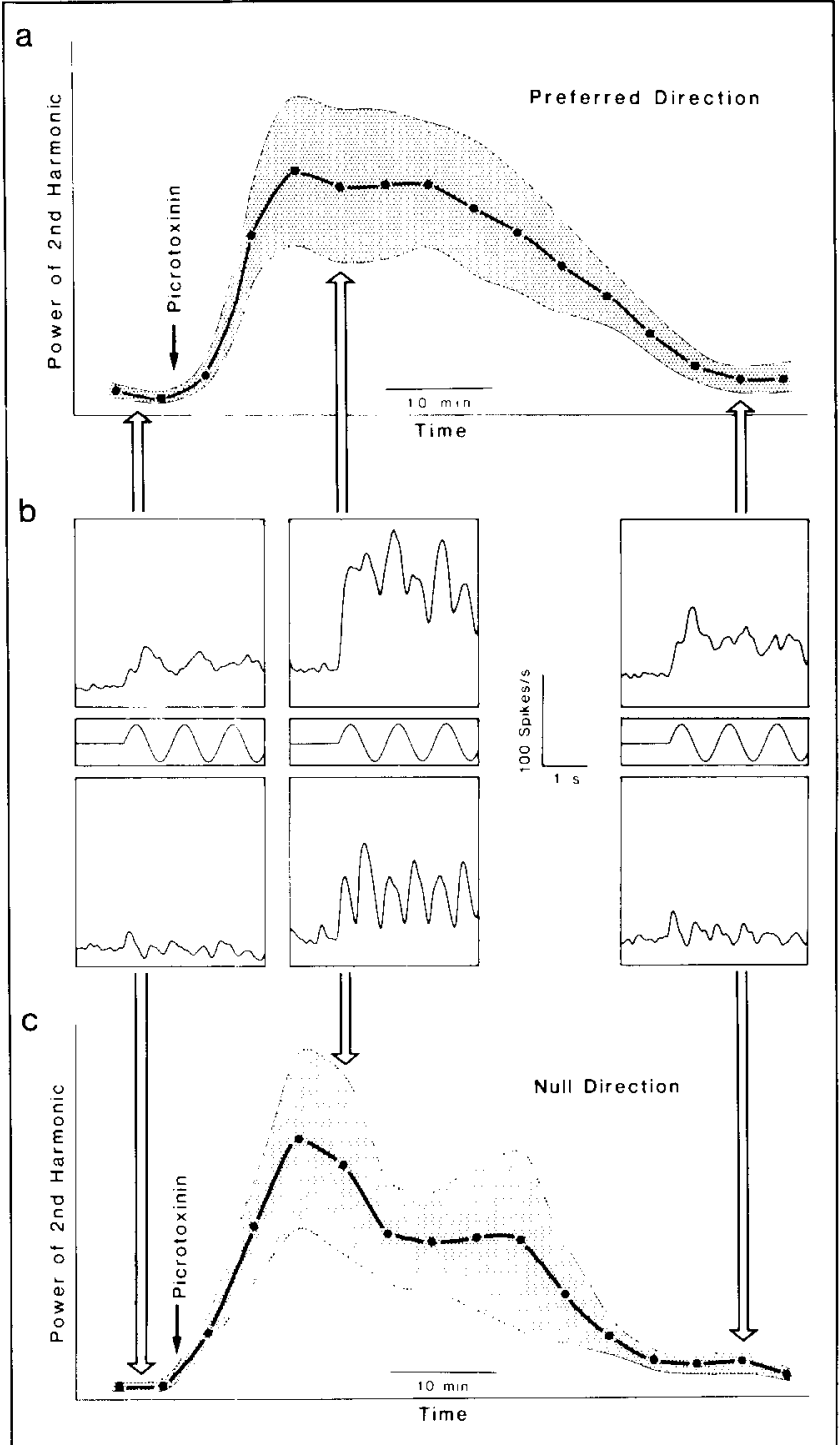

non-linearity of the shunting inhibition type. In contrast, both a suppression and an enhancement, with only their relative strength depending on the exact stimulus conditions, are an almost inevitable consequence of a twostage process of computing direction selectivity with a separate non-linear interaction and a subtraction stage.

1 Adelson, E.H. and Bergen, J.R., Spatiotemporal energy models for the perception of motion, J. Opt. Soc. Am. A, 2 (1985) 284-299.

2 Ariel, M. and Adolph, A.R., Neurotransmitter inputs to directionally sensitive turtle retinal ganglion cells, $J$. Neurophysicl., 54 (1985) 1123-1143.

3 Ariel, M. and Daw, N.W., Pharmacological analysis of directionally sensitive rabbit retinal ganglion cells, J. Physiol. (Lond.), 324 (1982) 161-185.

4 Barlow, H.B. and Levick, W.R., The mechanism of directionally selective units in rabbit's retina, $J$. Physiol. (Lond.), 178 (1965) $477-504$.

5 Benson, J.A., Transmitter receptors on insect neuronal somata: GABAergic and cholinergic pharmacology. In G.G. Lunt (Ed.),
Fig. 3. Responses of the Hl-cell in the fly's third visual ganglion to motion in its preferred and null direction before and after application of the GABA antagonist picrotoxinin $(250 \mathrm{nl}$ of a $1 \mathrm{mM}$ solution of picrotoxinin (Sigma) in Ringer ${ }^{24}$ ) into the fly's haemolymph next to the third visual ganglion. The stimulus consisted of a vertical sine-wave grating with a spatial wavelength of $68^{\circ}$, a mean luminance of $25 \mathrm{~cd} / \mathrm{m}^{2}$ and a contrast of 0.3 . The pattern was seen by the fly through a vertical slit with a size of $8.5^{\circ} \times 81^{\circ}$ which was located in front of the right eye at a horizontal angular position of $45^{\circ}$. The stimulus pattern was generated on a CRT-screen (Tektronix 608) by an image synthesizer (Picasso, Innisfree) which was controlled by programs written in ASYST on an IBM-AT computer. With this slit technique the eye is stimulated by only part of a spatial wavelength of the stimulus pattern. This allows to infer the properties of local movement detectors from the responses of spatially integrating neurones ${ }^{9}$. b: time course of the response of a $\mathrm{Hl}$-cell to motion in the preferred direction (i.e. from the back to the front; upper diagrams) and null direction (i.e. from the front to the back; lower diagrams); the middle diagrams show the brightness of the stimulus in the middle of the slit. Response traces are spike frequency histograms representing averages of 10 consecutive presentations of the stimulation program obtained in a single fly; motion in the preferred and null direction were presented alternately. The left diagrams show responses before injection of picrotoxinin; the middle and right diagrams show responses about $12 \mathrm{~min}$ and $55 \mathrm{~min}$ after injection. The responses are modulated in time; after injection of picrotoxinin the mean response and the amplitude of the response modulations increase. Note that after picrotoxinin injection the responses are mainly modulated with the second harmonic of the temporal frequency of stimulus motion. The changes induced by picrotoxinin are reversible. (a) and (c): the power of the second harmonic frequency component of the responses to motion in the preferred and null direction as a function of time, respectively. The power of the second harmonic was determined from consecutively obtained response profiles such as shown in (b) by 'Fast Fourier Analysis'. The data represent mean values and standard errors of the mean obtained from 10 flies. After picrotoxinin injection (injection time indicated by an arrow) the mean power of the second harmonic frequency component increases steeply for both motion in the cell's preferred and null direction and only returns to its preinjection level after approximately $40-50 \mathrm{~min}$.

Thus, the role of inhibition in motion detection systems other than the fly has to be reconsidered.

We would like to thank R. Hardie and A. Schmid for helpful advice concerning pharmacological problems, K.G. Götz, W. Reichardt, A. Schmid and J.M. Zanker for critical comments on the manuscript, and F. Buchstäber for skillfully drawing the figures.

Neurotox '88: Molecular Basis of Drug and Pesticide Action Elsevier, Amsterdam, 1988.

6 Borst, A. and Bahde, S., Processing of movement information in the fly's landing system: a behavioral analysis. In N. Singh and N. Strausfeld (Eds.), International Conference on Neurobiology of Sensory Systems, Plenum, New York, in press.

7 Borst, A. and Egelhaaf, M., Principles of motion detection, Trends Neurosci., 12 (1989) 297-306.

8 Caldwell, J.H., Daw, N.W. and Wyatt, H.J., Effects of picrotoxin and strychnine on rabbit retinal ganglion cells: lateral interactions of cells with more complex receptive fields, $J$. Physiol. (Lond.), 276 (1978) 277-298.

9 Egelhaaf, M., Borst, A. and Reichardt, W., Computational structure of a biological motion detection system as revealed by 
local detector analysis, J. Opt. Soc. Am. A, 6 (1989) 1070-1087.

10 Egelhaaf, M., Hausen, K., Reichardt, W. and Wehrhahn, C., Visual course control in flies relies on neuronal computation of object and background motion, Trends Neurosci., 11 (1988) 351-358.

11 Emerson, R.C. and Gerstein, G.L., Simple striate neurons in the cat. II. Mechanisms underlying directional asymmetry and directional selectivity, $J$. Neurophysiol., 40 (1977) 136-154.

12 Franceschini, N., Riehle, A. and Le Nestour, A., Directionally selective motion detection by insect neurons. In D. Stavenga and R. Hardie (Eds.), Facets of Vision, Springer, Berlin, 1989, pp. 360-390.

13 Ganz, L. and Felder, R., Mechanism of directional selectivity in simple neurons of the cat's visual cortex analyzed with stationary flash sequences. J. Neurophysiol., 51 (1984) 294-324.

14 Grzywacz, N.M. and Koch, C., Functional properties of models for direction selectivity in the retina, Synapse, 1 (1987) 417-434.

15 Hausen, K., The lobula-complex of the fly: structure, function and significance in visual behaviour. In M.A. Ali (Ed.), Photoreception and Vision in Invertebrates, Plenum, New York, 1984 , pp. 523-559

16 Koch, C., Poggio, T. and Torre, V., Computations in the vertebrate retina: gain enhancement, differentiation and motion discrimination, Trends Neurosci., 9 (1986) 204-211.

17 Levick, W.R., Oyster, C.W. and Takahashi, E., Rabbit lateral geniculate nucleus: sharpener of directional information, $S \mathrm{ci}$ ence, 165 (1969) 712-714.

18 Marchiafava, P.L., The organization of inputs establishes two functional and morphologically identifiable classes of ganglion cells in the retina of the turtle, Vis. Res., 23 (1983) 325-338.

19 Mikami, A., Newsome, W.T. and Wurtz, R.H., Motion selectivity in macaque visual cortex. I. Mechanisms of direction and speed selectivity in extrastriate area MT, J. Neurophysiol., 55
(1986) $1308-1327$.

20 Movshon, J.A., Thompson, I.D. and Tolhurst, D.J., Receptive field organization of complex cells in the cat's striate cortex, $J$. Physiol. (Lond.), 283 (1978) 79-99.

21 Reichardt, W., Autocorrelation, a principle for the evaluation of sensory information by the central nervous system. In W.A Rosenblith (Ed.), Sensory Communication, MIT/Wiley, New York, 1961, pp. 303-317.

22 Reichardt, W., Evaluation of optical motion information by movement detectors, J. Comp. Physiol. A, 161 (1987) 533-547.

23 Van Santen, J.P.H. and Sperling, G., Elaborated Reichardt detectors, J. Opt. Soc. Am. A, 2 (1985) 300-321.

24 Schmid, A. and Bülthoff, H., Using neuropharmacology to distinguish between excitatory and inhibitory movement detection mechanisms in the fly, Calliphora erythrocephala, Biol. Cybern., 59 (1988) 71-80.

25 Sillito, A.M., Inhibitory processes underlying the directional specificity of simple, complex and hypercomplex cells in the cat's visual cortex, J. Physiol. (Lond.), 271 (1977) 699-720.

26 Srinivasan, M.V. and Bernard, G.D., A proposed mechanism for multiplication of neural signals, Biol. Cybern., 21 (1976) 227-236.

27 Torre, V. and Poggio, T., A synaptic mechanism possibly underlying directional selectivity to motion, Proc. R. Soc. Lond. Ser. B., 202 (1978) 409-416.

28 Werblin, F., Maguire, G., Lukasiewicz, P., Eliasof, S. and Wu S.M., Neural interactions mediating the detection of motion in the retina of the tiger salamander, Vis. Neurosci., 1 (1988) 317-329.

29 Wyatt, H.J. and Daw, N.W., Specific effects of neurotransmitter agonists on ganglion cells in rabbit retina, Science, 191 (1976) 204-205 